\title{
Control of the properties of porous chitosan-alginate membranes through the addition of different proportions of Pluronic F68
}

\author{
Cecilia Zorzi Bueno ${ }^{a}$, Ana Maria Antunes Dias ${ }^{b}$, Hermínio José Cipriano de Sousa ${ }^{\text {b }}$, \\ Mara Elga Medeiros Braga ${ }^{b}$, Ângela Maria Moraes ${ }^{a, *}$ \\ a Department of Engineering of Materials and of Bioprocesses, School of Chemical Engineering, University of Campinas (UNICAMP), Campinas, SP CEP 13083-852, Brazil

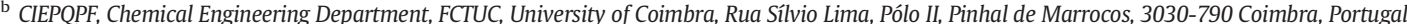

\section{A R T I C L E I N F O}

\section{Article history:}

Received 10 April 2014

Received in revised form 27 June 2014

Accepted 3 August 2014

Available online 10 August 2014

\section{Keywords:}

Alginate

Chitosan

Surfactant

Scaffold

Wound dressing

\begin{abstract}
A B S T R A C T
This work addresses the development and characterization of porous chitosan-alginate based polyelectrolyte complexes, obtained by using two different proportions of the biocompatible surfactant Pluronic F68. These biomaterials are proposed for applications as biodegradable and biocompatible wound dressing and/or scaffolds. The results indicate that thickness, roughness, porosity and liquid uptake of the membranes increase with the amount of surfactant used, while their mechanical properties and stability in aqueous media decrease. Other important properties such as color and surface hydrophilicity (water contact angle) are not significantly altered or did not present a clear tendency of variation with the increase of the amount of surfactant added to the polyelectrolyte complexes, such as real density, average pore diameter, total pore volume and surface area. The prepared biomaterials were not cytotoxic to L929 cells. In conclusion, it is possible to tune the physicochemical properties of chitosan-alginate polyelectrolyte complexes, through the variation of the proportion of surfactant (Pluronic F68) added to the mixture, so as to enable the desired application of these biomaterials.
\end{abstract}

(c) 2014 Elsevier B.V. All rights reserved.

\section{Introduction}

Polysaccharides are biopolymers that have attracted great interest in the medical and scientific communities due to their interesting properties which include biodegradability, biocompatibility, relatively low manufacturing and disposal costs, environmentally friendly aspects and renewability prospect. This class of materials is proving to be very versatile, with a wide variety of biomedical applications that range from topical wound dressings to scaffolds in the tissue engineering field [1].

An ideal wound dressing should protect the wound bed from penetration of microorganisms and external threats, maintain a moist healing environment (while removing excess exudate), allow oxygen permeation, improve healing with minimal scar formation, be easy to apply and remove, and be of low cost. Films and hydrogels made of polysaccharides are frequently investigated for this purpose [2-4]. Biodegradable wound dressings, with or without cultured cells, may also function as skin substitutes $[1,5]$. These dressings may be able to

\footnotetext{
* Corresponding author. Tel.: +55 1935213920.

E-mail addresses: ceciliazb@feq.unicamp.br (C.Z.Bueno), adias@eq.uc.pt (A.M.A. Dias), hsousa@eq.uc.pt (H.J.C. de Sousa), marabraga@eq.uc.pt (M.E.M. Braga), ammoraes@feq.unicamp.br (Â.M. Moraes).
}

interact with the surrounding tissues, and stimulate cell migration, angiogenesis, and epithelialization and promote accelerated wound healing, through the provision of an adequate scaffold for new tissue development $[1,5]$. In these cases, the scaffold should have an adequate three-dimensional porous structure, with high surface area to provide a suitable environment to which cells could adhere, migrate and grow, as well as mechanical properties similar to those of the injured tissue. The scaffold should also degrade into nontoxic components that could be eliminated or reabsorbed by the organism, and ideally its degradation rate should match the rate of new tissue formation $[1,6]$. Several methodologies are used nowadays to produce porous scaffolds from biopolymers, such as gas foaming, freeze-drying, solvent casting and particulate leaching, 3D printing, or combinations of these methods [6].

The polysaccharides most commonly used for the development of wound dressings and scaffolds for bone, cartilage, and skin tissue regeneration include chitin and chitosan, alginate, hyaluronic acid, starch and cellulose-based polymers and their derivatives, as recently reviewed by Khan and Ahmad [1]. Xanthan gum has also drawn interest, mostly when combined with chitosan $[7,8]$.

Chitosan-alginate based polyelectrolyte complexes (PEC) have been thoroughly investigated by many research groups over the last years [9-18] due to their higher stability to changes in the $\mathrm{pH}$ of the medium and improved effectiveness as controlled-release systems and when compared to chitosan or alginate alone [1]. The pioneer efforts of Yan et al. [15] were followed by those of Wang et al. [16], who developed 
a chitosan-alginate PEC that was further cross-linked with $\mathrm{Ca}^{2+}$ ions. This material presented adequate flexibility and stability in water. A few years later, Rodrigues et al. [19] proposed a modification of the methodology established by Wang et al. [16] with significantly better prospects for scale up. Later, Bueno and Moraes [20] introduced changes in the process aiming to produce porous chitosan-alginate PEC membranes, through the addition of biocompatible surfactants, such as Pluronic F68, to the PEC. Pluronic F68 is a non-ionic surfactant frequently used in the pharmaceutical industry due to its compatibility with various drugs and excipients, being approved by the FDA as a component of skin products. A study done with more than 1000 patients with dermal lesions demonstrated that the application of Pluronic F68 to the injuries did not cause adverse reactions and also prevented the occurrence of infections [21]. The porous membranes obtained with the addition of Pluronic F68, were produced using a simple and inexpensive approach, which is not based on freeze-drying or other vacuum based protocols, being therefore considered as potential candidates for biomedical applications, such as wound dressings that can be used directly on skin lesions or as scaffolds for cell culture that can be later transferred to the injured region.

However, the surfactant/polysaccharide ratio used was considered high and able to induce toxicity to skin cells. Therefore, the present work aimed to improve the previously reported formulation and manufacturing process through the reduction of the amount of surfactant Pluronic F68 and organic solvents used and time consumed during the PEC drying step. The obtained biomaterials were thoroughly characterized in order to conclude about their applicability as wound dressings and scaffolds for tissue engineering.

\section{Materials and methods}

\subsection{Chemicals}

Chitosan from shrimp shells (deacetylation degree of $88 \%$, MW of $1.26 \times 10^{6} \mathrm{~g} / \mathrm{mol}$, intrinsic viscosity of $848 \mathrm{~mL} / \mathrm{g}$ at $25^{\circ} \mathrm{C}$ ) and low viscosity sodium alginate (MW of $4.69 \times 10^{4} \mathrm{~g} / \mathrm{mol}$, intrinsic viscosity of $366 \mathrm{~mL} / \mathrm{g}$ at $25^{\circ} \mathrm{C}$ ) were both from Sigma-Aldrich (United States). The deacetylation degree of chitosan was provided by Sigma-Aldrich and the molar masses and intrinsic viscosities were determined by viscosimetry (using a capillary viscometer Ostwald-Cannon-Fenske, size 200) and using the constants of the Mark-Houwink-Sakurada equation from Mancini et al. and Canella and Garcia [22,23].

Pluronic F68, phosphate buffered saline (PBS), Hepes buffer, $\mathrm{NaCl}$, sodium dodecyl sulfate (SDS), dimethyl sulfoxide (DMSO) and 3-(4,5dimethylthiazol-2-yl)-2,5-diphenyltetrazolium bromide (MTT) were also obtained from Sigma-Aldrich (United States). Fetal bovine serum and RPMI-1640 cell culture medium were obtained from Nutricell (Brazil). Calcium chloride dihydrate and sodium hydroxide were obtained from Merck (Germany) and acetic acid was obtained from Synth (Brazil). All reagents were of at least analytical grade quality and/or suitable for cell culture.

\subsection{Preparation of the membranes}

Membranes were prepared based on modifications of the process previously reported by Bueno and Moraes [20]. The proportion of surfactant Pluronic F68 added to the polysaccharide mixture was reduced from $1: 1(\mathrm{w} / \mathrm{w})$ to $0.02: 1$ and $0.1: 1(\mathrm{w} / \mathrm{w})$, to analyze the effect of Pluronic F68 on the physicochemical properties of the membranes when used at lower surfactant/polysaccharide ratios. Acetone (previously used) was eliminated from the chitosan solution and the PEC drying temperature was increased from $37{ }^{\circ} \mathrm{C}$ to $60{ }^{\circ} \mathrm{C}$. These changes are intended to reduce the cytotoxic potential of residual surfactant (that may persist even after the successive washing steps done during the preparation of the membranes), to decrease production costs and to speed up the production of the membranes.

The experimental procedure consists in the addition of $90 \mathrm{~mL}$ of $1 \%(\mathrm{w} / \mathrm{v})$ chitosan dissolved in $2 \%(\mathrm{v} / \mathrm{v})$ aqueous acetic acid solution to $180 \mathrm{~mL}$ of an aqueous solution of alginate at $0.5 \%(\mathrm{w} / \mathrm{v})$ containing $0.02 \%$ or $0.1 \%(\mathrm{w} / \mathrm{v})$ of Pluronic F68 using a flow rate of $200 \mathrm{~mL} / \mathrm{h}$ (using a peristaltic pump Minipuls 3, Gilson). The mixture occurred in a jacketed stainless steel tank with internal diameter of $10 \mathrm{~cm}$ and height of $20 \mathrm{~cm}$ with temperature controlled at $25{ }^{\circ} \mathrm{C}$ (thermosthatic bath Q-214M2, Quimis) and stirred at $500 \mathrm{rpm}$ (using a mechanical stirrer model Q-251D, Quimis, with a $4 \mathrm{~cm}$ in diameter three tilted-blade propeller). The final suspension was homogenized at $1000 \mathrm{rpm}$ during $10 \mathrm{~min}$. The $\mathrm{pH}$ of the suspension was increased to 5.3 with an aqueous solution of $\mathrm{NaOH}$ at $1 \mathrm{M}[19,24]$ and the same stirring rate was maintained for $10 \mathrm{~min}$. Then, $3.6 \mathrm{~mL}$ of a $2 \%(\mathrm{w} / \mathrm{v}) \mathrm{CaCl}_{2}$ aqueous solution was added to the mixture to crosslink the alginate carboxyl groups not linked to chitosan. The volume and concentration of the $\mathrm{CaCl}_{2}$ solution were previously optimized to ensure the formation of a membrane with adequate properties $[16,19]$. The solution was further stirred for $10 \mathrm{~min}$. The mixture was transferred to polystyrene Petri dishes (15 cm in diameter) and casted at $60{ }^{\circ} \mathrm{C}$ in an oven (model $410 \mathrm{D}$, Nova Ética) for $13 \mathrm{~h}$. For further crosslinking, the membranes were immersed in $150 \mathrm{~mL}$ of a $2 \%(\mathrm{w} / \mathrm{v}) \mathrm{CaCl}_{2}$ aqueous solution for $30 \mathrm{~min}$. The membranes were then washed for 30 min according to the following sequence: two immersions in $200 \mathrm{~mL}$ of deionized water, one immersion in $250 \mathrm{~mL}$ of $10 \mathrm{mM}$ Hepes buffer and again in $500 \mathrm{~mL}$ of deionized water. Finally the membranes were dried at $37^{\circ} \mathrm{C}$ for $6 \mathrm{~h}$ (with their edges fixed to the borders of the Petri dishes to avoid shrinking).

Membranes prepared without surfactant Pluronic F68 were also prepared as control. The preparation of these membranes required a further step, which consisted in the deaeration of the mixture under vacuum for 120 min with the aid of a vacuum pump (model TE-058, Tecnal) before drying. The remaining steps of the preparation procedure were performed as previously described for the membranes with Pluronic F68. The samples were identified according to the Pluronic F68 proportion over the polysaccharide mass as P0\% (without surfactant), P2\% and P10\%.

\subsection{Characterization of the membranes}

The samples used to characterize properties that are influenced by humidity, such as morphology, fluid handling properties and mechanical properties, were stored in a desiccator with silica gel at room temperature for at least $48 \mathrm{~h}$ before analyses. For the other characterization methods, the samples were stored at $20 \%$ relative humidity at room temperature for at least $48 \mathrm{~h}$ before analysis.

\subsubsection{FTIR-ATR spectroscopy}

FTIR-ATR spectroscopy (Jasco, model 4200) was performed at 128 scans with a $4 \mathrm{~cm}^{-1}$ resolution between 500 and $4000 \mathrm{~cm}^{-1}$ and using a Golden Gate Single Reflection Diamond ATR accessory. The spectra obtained for the membranes were compared to those of the pure polymers which were analyzed in the form of dry powders.

\subsubsection{Aspect}

The aspect of the samples was evaluated based on their morphology, porosity, thickness, roughness and color.

The morphology of the samples was evaluated macroscopically (using a photographic camera model DMC-F2, Panasonic) and microscopically (using an optical microscope, Olympus BH-2) and with a scanning electron microscope model LEO 440, Leica operating at $10 \mathrm{kV}$ and $50 \mathrm{pA}$. Before the scanning electron microscopy (SEM) analysis, the samples were coated with an ultra-thin layer of gold $(92 \AA)$ in a mini sputter coater (SC 7620, VG Microtech).

The microporosity of the films was measured by $\mathrm{N}_{2}$ adsorption (Micrometrics ASAP 2000 V2.04.). The surface area was calculated 
with the BET equation. The total porosity was calculated according to Eq. (1):

$P=1-\rho_{\text {apparent }} / \rho_{\text {solid }}$

where $\rho_{\text {apparent }}\left(\mathrm{g} / \mathrm{cm}^{3}\right)$ is the apparent density (calculated as the ratio between the mass and the volume of each sample), and $\rho_{\text {solid }}\left(\mathrm{g} / \mathrm{cm}^{3}\right)$ is the true density measured by helium pycnometry (Accupyc 1330 Micromeritics, USA).

The thickness of the membranes was determined using a digital micrometer (model MDC-25S, Mitutoyo) and is reported as an average of 10 measurements at different positions of each sample.

The roughness of the membranes (expressed as the arithmetical mean roughness, $R a$ ) was evaluated using a digital rugosimeter (model SJ-201, Mitutoyo) and is reported as an average of 10 measurements at different positions of each sample. The cutoff wavelength and total evaluation length were defined before measurements and chosen according to ISO 4288 (1996) and ASME B46.1 (2002) specifications $[25,26]$.

The color of the membranes was evaluated in triplicate according to the CIELAB color space, using a portable colorimeter (model CT-310, Minolta). Luminosity and color were determined by $L, a$ and $b$ parameters. The parameter $L$ varies from 0 (black) to 100 (white), $a$ varies from -60 (green) to +60 (red) and $b$ varies from -60 (blue) to +60 (yellow). Those parameters were used to calculate the tonality (Hue), chroma metric $\left(C_{M}\right)$ and color differences between membranes prepared with and without Pluronic F68 $(\Delta E)$ according to Eqs. (2) to (4), respectively:

Hue $=\arctan (b / a)$

$C_{M}=\sqrt{a^{2}+b^{2}}$

$\Delta E=\sqrt{\Delta L^{2}+\Delta a^{2}+\Delta b^{2}}$

\subsubsection{Behavior in aqueous media}

Liquid uptake was measured in water, $0.9 \% \mathrm{NaCl}$, PBS, fetal bovine serum (FBS) and simulated body fluid (SBF) (prepared according to Kokubo et al. [27]). The samples $(6 \times 1, \mathrm{~cm})$ were immersed in $10 \mathrm{~mL}$ of each solution for $24 \mathrm{~h}$ at $37^{\circ} \mathrm{C}$. Liquid uptake $(U)$ was measured in triplicate according to Eq. (5), where $W_{w}$ is the weight of the wet sample and $W_{d}$ is the weight of the dry sample.

$U=\left(W_{w}-W_{d}\right) / W_{d}$

Mass loss was measured using rectangular samples $(6 \times 1, \mathrm{~cm})$ immersed in $10 \mathrm{~mL}$ of each solution for 7 days at $37^{\circ} \mathrm{C}$. After this period, they were further washed repeatedly (5 times) in $20 \mathrm{~mL}$ of deionized water for $5 \mathrm{~min}$, to remove salts, proteins, sugars and ions, as well as unbounded polysaccharide chains. The membranes were then dried at $37{ }^{\circ} \mathrm{C}$ for at least $72 \mathrm{~h}$ until they reach constant weight. The mass loss $(M)$ of the membranes was calculated according to Eq. (6), in which $W_{i}$ is the initial weight and $W_{f}$ is the final weight of the samples after immersion in the different solutions.

$M=\left(W_{i}-W_{f}\right) / W_{i} \times 100$

Static water contact angle measurements were performed using OCA20 equipment (Dataphysics). Due to the porous and irregular surfaces of the films, the samples were previously compacted with a manual press for $1 \mathrm{~min}$. Twenty measurements were made for each type of sample (P0\%, P2\% and P10\%).

\subsubsection{Mechanical properties}

Tensile strength and elongation at break were determined using rectangular samples $(10 \mathrm{~cm} \times 2.54 \mathrm{~cm})$ according to the ASTM D882
(1995) standard [28] using a texturometer (model TA.XT2, Stable Microsystems SMD) and employing a crosshead speed of $0.1 \mathrm{~cm} / \mathrm{s}$ and gauge length of $5 \mathrm{~cm}$. Tensile strength (TS) and elongation at break $(E B)$ were calculated according to Eqs. (7) and (8), respectively, in which $S_{m}$ is the maximum strength at break, $A_{s}$ is the cross section area of the sample, $d_{i}$ is the initial distance between the texturometer grips $(5 \mathrm{~cm})$ and $d_{b}$ is the distance between the grips right before the break of each sample.

$T S=S_{m} / A_{S}$

$E B=\left(d_{b}-d_{i}\right) / d_{i} \times 100$

\subsubsection{Indirect cytotoxicity}

Indirect in vitro cytotoxicity was evaluated according to the ISO 10993-5e (1992) standard [29] using nearly confluent monolayer L929 fibroblasts cultivated in supplemented RPMI-1640 medium and 3-(4,5-dimethylthiazol-2-yl)-2,5-diphenyltetrazolium bromide (MTT). In this colorimetric assay, MTT is cleaved only by live and metabolically active cells, forming a blue precipitate (formazan) which is quantified spectrophotometrically at $620 \mathrm{~nm}$. Membrane extracts were obtained by incubating the samples in RPMI-1640 culture medium supplemented with Hepes buffer and fetal bovine serum, at a concentration of $0.05 \mathrm{~g}$ of dry material per milliliter of medium for $48 \mathrm{~h}$ at $37{ }^{\circ} \mathrm{C}$ and a $5 \% \mathrm{CO}_{2}$ atmosphere. Aliquots of $100 \mu \mathrm{L}$ of a cell suspension in culture medium containing $1 \times 10^{5}$ cells $/ \mathrm{mL}$ were transferred to 96 -well flat bottom plates (brand TPP, Switzerland) and maintained at $37{ }^{\circ} \mathrm{C}$ and $5 \% \mathrm{CO}_{2}$ for $24 \mathrm{~h}$. Afterwards, the medium was removed and $100 \mu \mathrm{L}$ of the solution in which the membranes were immersed (extract of the samples) was added to each well, and cells were incubated for a further $24 \mathrm{~h}$ period. After that period, the extracts were removed, the wells were washed twice with $100 \mu \mathrm{L}$ of PBS/EDTA and $100 \mu \mathrm{L}$ of culture medium and $10 \mu \mathrm{L}$ MTT at $5 \mathrm{mg} / \mathrm{mL}$ in PBS/EDTA were added to each well and the plates were again incubated at $37{ }^{\circ} \mathrm{C}$ and $5 \% \mathrm{CO}_{2}$ for $4 \mathrm{~h}$. Then, $100 \mu \mathrm{L}$ of sodium dodecyl sulfate at $100 \mathrm{~g} / \mathrm{L}$ in dimethyl sulfoxide containing $0.6 \%(\mathrm{v} / \mathrm{v})$ acetic acid was added to each well. Samples were gently homogenized and the cultures were returned to incubation at $37{ }^{\circ} \mathrm{C}$ for $1 \mathrm{~h}$. The absorbance of each well was measured at $620 \mathrm{~nm}$ using an ELISA microplate spectrophotometer (Multiskan FC, Thermo Scientific). Latex and fragments of the culture plate exposed to culture medium were used as positive and negative controls respectively and culture medium without cells was used as blank. The experiments were performed at least in quintuplicates.

\section{Results and discussion}

The present work addresses improvements in the manufacturing process of porous chitosan-alginate based wound dressings and/or scaffolds aiming cost, chemical, processing time and potential toxicity reduction. The physical-chemical-mechanical and biological properties of different biomaterials, prepared without and with two amounts of surfactant (Pluronic F68), are compared and discussed as follows.

\subsection{FTIR-ATR spectroscopy}

This technique was used to evaluate the presence of residual Pluronic F68 in the samples prepared with the surfactant and the formation of chitosan-alginate polyelectrolyte complex (PEC). The spectra of pure and non-processed chitosan, alginate and Pluronic F68, obtained directly from these reagents in the form of powders, and of the membranes prepared without and with different Pluronic F68 proportions are shown in Fig. 1, while the characteristic bands of each compound are given in Table 1.

The characteristic bands of both polysaccharides are clearly identified, such as the broad absorption band between 3600 and $3000 \mathrm{~cm}^{-1}$ for both chitosan and alginate, attributed to $-\mathrm{OH}$ stretching [33]. 


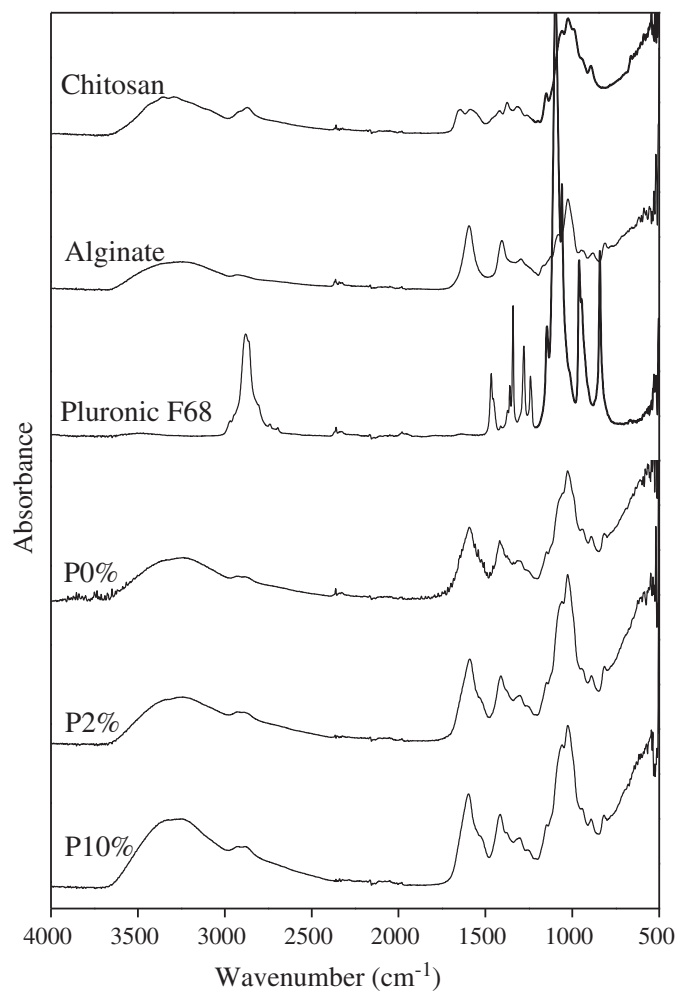

Fig. 1. FTIR-ATR absorption spectra of pure chitosan, alginate, Pluronic F68 and of the membranes prepared without and with different Pluronic F68 proportions.

Chitosan and alginate showed also a band at approximately $2900 \mathrm{~cm}^{-1}$, characteristic of methyl and methylene vibrations [32-34]. This band was also noticeable in the Pluronic F68 spectrum.

The interaction between alginate carboxyl groups and chitosan amino groups results in a broad absorption band between 1560 and $1640 \mathrm{~cm}^{-1}$ [33]. Therefore, it is difficult to identify the presence of free chitosan and alginate as well as of their protonation state in the absorption spectra of the films, what would be essential to confirm the formation of the PEC.

Lawrie et al. [33] also observed that the FTIR absorption spectra of different types of chitosan and alginate mixtures, such as precipitates, dense films and alternated layer films, are very similar, despite the diverse interaction intensities between the polysaccharide functional groups in each case. In this sense, the FTIR technique is somehow limited to confirm the PEC formation. The presence of residual Pluronic F68 in the membranes could not be confirmed by the FTIR-ATR technique.

\subsection{Aspect of the membranes}

The aspect of the samples prepared with and without surfactant was analyzed and compared in terms of their morphology, thickness, rugosity, porosity and color. The typical morphology of the membranes is shown in Fig. 2, while the results of porosity are reported in Table 2

Table 1

Characteristic FTIR absorption bands for pure chitosan, alginate and Pluronic F68.

\begin{tabular}{llll}
\hline Band $\left(\mathrm{cm}^{-1}\right)$ & Group & Compound & Reference \\
\hline 1098 & Aliphatic ether & Pluronic F68 & {$[30]$} \\
1150 & Aliphatic ether & Pluronic F68 & {$[31]$} \\
1405 & $-\mathrm{COOH}$ & Alginate & {$[32]$} \\
1560 & $-\mathrm{NH}$ & Chitosan & {$[32]$} \\
1580 & $-\mathrm{NH}_{2}$ & Chitosan & {$[32]$} \\
1600 & $-\mathrm{COOH}$ & Alginate & {$[32]$} \\
1650 & $-\mathrm{C}=\mathrm{O}$ from amide group & Chitosan & {$[32]$} \\
\hline
\end{tabular}

and those of rugosity, thickness and color parameters are indicated in Table 3.

\subsubsection{Morphology}

The morphological aspect of the chitosan-alginate membranes changed substantially with the inclusion of the surfactant Pluronic F68. This effect can be explained by the high hydrophilic-lipophilic balance (HLB) value of Pluronic F68, which is equal to 29 [35], indicating that this surfactant is highly soluble in water and can therefore form foams (as previously reported by Bueno and Moraes [20]). The higher amount of surfactant employed in the preparation of the membranes led to higher air retention during the mechanical stirring, with a consequent increase in the pore size of the biomaterials.

As can be confirmed in Fig. 2B (images obtained by optical microscopy) sample $\mathrm{P} 10 \%$ presents the largest pore size cavities. Moreover, some of those pores present rupture of their walls, which probably indicates burst during the drying step in the oven with forced air circulation. As observed by SEM (Fig. 2C), those pores do not cross the whole thickness of the membranes which present a lamellar structure. This characteristic favors the application of the biomaterial in the treatment of skin lesions, since it avoids the penetration of microorganisms into the wound $[20,36]$.

It is believed that Pluronic F68 interacts with chitosan and alginate mostly through hydrophilic interactions, due to the high - OH content, which induces an extended conformation of the polymers in solution [37]. Considering the hydrophilic/hydrophobic character of Pluronic F68, it can be assumed that those molecules must be oriented with their polar chains (two polyoxyethylene chains) interacting with water or with the polysaccharides, and with their non-polar chain (one polyoxypropilene chain) interacting with the air trapped inside the pore. In this way, the presence of the surfactant molecules permits us to maintain the structural integrity of the membrane as water evaporates from the liquid thin film that constitutes the foam. It can be assumed that this high stability is mainly due to the strong association between the macromolecules and due to the high viscosity of the system, among other factors. The strong association between Pluronic F68 and alginate was previously studied by Stoppel et al. [38]. The authors reported that Pluronic F68 and alginate chains may form a complex, which increases the effective volume fraction of the polymer in water, leading to an increase in solution dynamic viscosity. The addition of $5 \%(\mathrm{w} / \mathrm{v})$ of Pluronic F68 to a $1 \%(\mathrm{w} / \mathrm{v})$ alginate solution increases the dynamic viscosity by $59 \%$ and increases the size of the alginate-Pluronic F68 complex by $110 \%$ [38]. The stability of the system may also be attributed to the decrease in the interfacial tension during water evaporation, as the concentration of Pluronic F68 increases [37]. Furthermore, water drainage was hampered by the presence of Pluronic F68, since this surfactant is believed to reduce drainage in foams [39].

\subsubsection{Porosity}

Membranes' porosity results are shown in Table 2 . The overall porosity of the samples was shown to increase from $~ 39$ up to $83 \%$ for samples $\mathrm{P} 2 \%$ and $\mathrm{P} 10 \%$, respectively which is in agreement with the morphology results previously discussed. The obtained data indicate that an increase in the membrane's surface area and total pore volume was observed only for sample P10\% (highest amount of surfactant used). Sample P2\% presented the lowest surface area and total pore volume compared to the other samples. Conversely, sample P2\% presented the largest average pore diameter. Literature recommends that for fluid management and dermal delivery applications the surface area of the dressings must range between 5 and $100 \mathrm{~m}^{2} / \mathrm{g}[41,42]$ and therefore samples $\mathrm{P} 0 \%$ and $\mathrm{P} 10 \%$ would be recommended for these applications.

The results obtained by He pycnometry show that sample P0\% was denser followed by samples P10\% and P2\%, in this order. According to Stoppel et al. [38], alginate and Pluronic gels shrink upon gelation with divalent ions. This shrinkage increases with the Pluronic F68 concentration, resulting in a decrease in the final hydrogel volume and in 

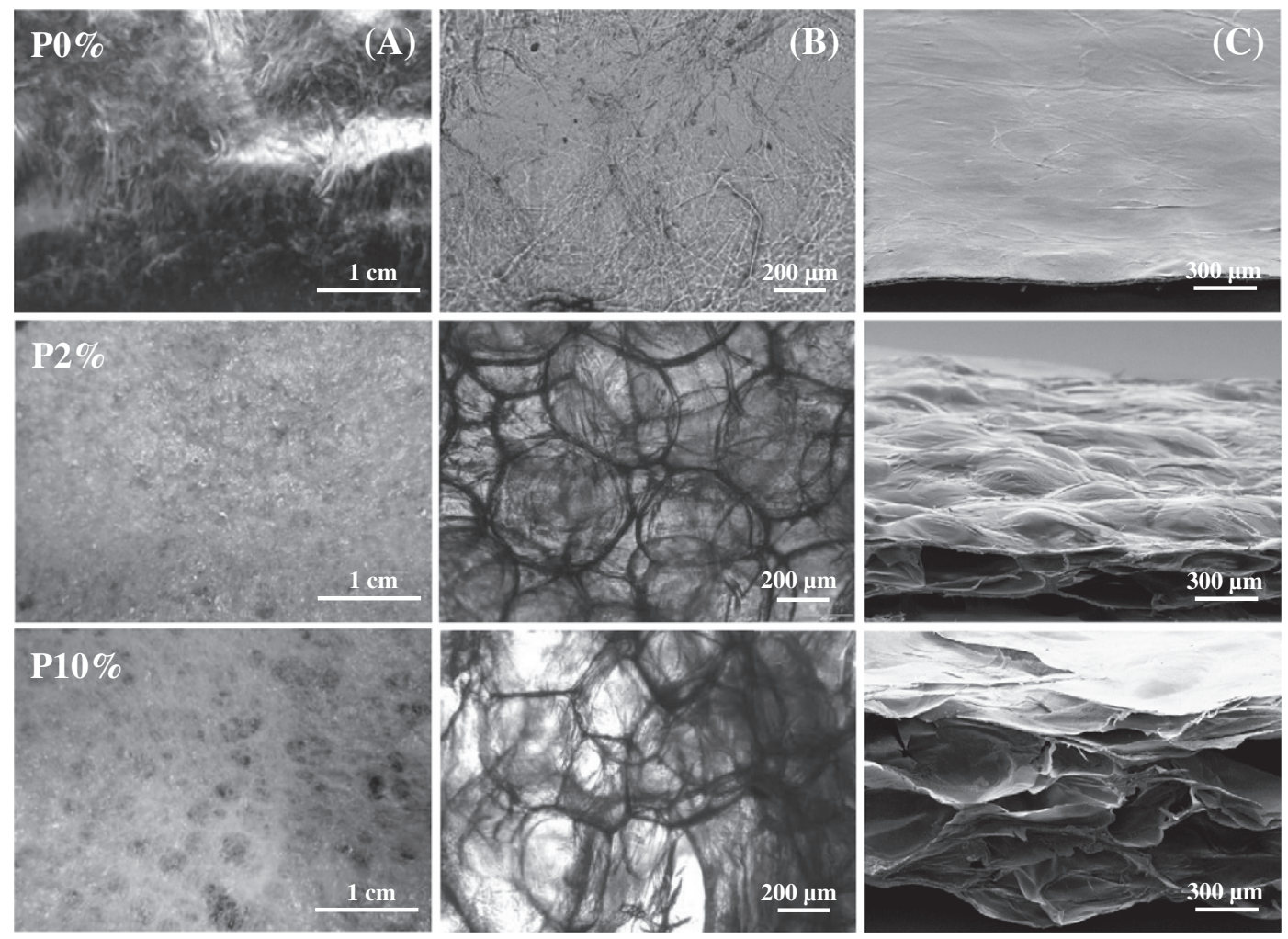

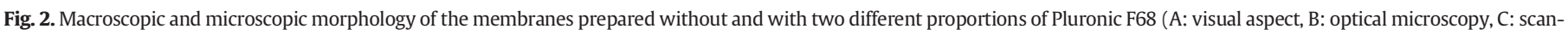
ning electron microscopy).

an increase in the final cross-linking density [38]. Therefore, Pluronic F68 could have an effect on the real density of the chitosan-alginate membranes, which was observed only for sample P10\%.

According to the porosity results, it can be concluded that samples P2\% and P10\% have potential application not only as wound dressings but also as scaffolds since they have a porous structure. According to Ribeiro et al. [6], scaffolds should have a porous structure, with high surface area, to allow for maximum cell loading and cell-matrix interactions, and to improve the transport of nutrients and oxygen into and out of the matrix. On the other hand, the denser and transparent characteristics of the membranes prepared without Pluronic F68 make them attractive to be used as wound dressings, since they would allow monitoring the healing process without the need to remove the dressing.

It is important to stress out that $\mathrm{N}_{2}$ adsorption data refers to the microporosity of the membranes, indicating the presence of a higher amount of micropores in sample P10\%, followed by sample P0\% and P2\%. Larger pore sizes (higher than $1000 \AA$ ) were not accounted in this analysis. However, He pycnometry accounts for the total free volume of the material, giving a reliable result regarding its real density. A more accurate result would be obtained by intrusion mercury porosimetry, which accounts for macropores, however this technique requires the use of high pressures which may lead to inaccurate results

Table 2

Analysis of the porosity by nitrogen adsorption and helium pycnometry of the membranes prepared in the presence of different Pluronic F68 amounts.

\begin{tabular}{llllll}
\hline Samples & \multicolumn{3}{l}{ BET method } & $\begin{array}{l}\text { Real density } \\
\left(\mathrm{g} / \mathrm{cm}^{3}\right)\end{array}$ & $\begin{array}{l}\text { Total } \\
\text { porosity }\end{array}$ \\
\cline { 2 - 5 } & $\begin{array}{l}\text { Surface area } \\
\left(\mathrm{m}^{2} / \mathrm{g}\right)\end{array}$ & $\begin{array}{l}\text { Total pore } \\
\text { volume } \\
\left(\mathrm{cm}^{3} / \mathrm{g}\right)\end{array}$ & $\begin{array}{l}\text { Average pore } \\
\text { diameter }(\mathrm{A})\end{array}$ & & \\
\hline P0\% & 5.63 & 0.0067 & 47.3 & $0.947 \pm 0.013$ & $0.46 \pm 0.02$ \\
P2\% & 1.04 & 0.0015 & 55.7 & $0.252 \pm 0.002$ & $0.64 \pm 0.02$ \\
P10\% & 7.67 & 0.0098 & 51.7 & $0.573 \pm 0.003$ & $0.84 \pm 0.04$ \\
\hline
\end{tabular}

in the case of flexible or fragile solids, such as polymers and aerogels [40].

\subsubsection{Thickness, roughness and color}

The analysis of the results obtained for the thickness and roughness of the membranes (Table 3) shows that an increase in the Pluronic F68 proportion leads to a significant increase in both properties, up to 6 and 17 times, respectively. An increase in thickness was expected since more air can be stably incorporated into the larger cavities that are formed when using a higher amount of surfactant, as previously discussed. This higher incorporation of air into the structure of the membranes certainly contributed to the simultaneous increase of the roughness of the samples.

According to Ma et al. [43], in vivo biodegradable dermis temporary substitutes should ideally be thinner than the human dermis, whose thickness varies from 0.5 up to $2 \mathrm{~mm}$ depending on age, sex and body region where the biomaterial is to be applied. The use of scaffolds with thickness lower than $1 \mathrm{~mm}$ has been described in the literature for the regeneration of human skin [44], which indicates that the membranes produced in this work can be considered adequate for this purpose.

The surface roughness of wound dressings and scaffolds affects cell adhesion and proliferation capacity, as well as the morphology of the cells cultivated on the surface of those biomedical devices. Roughness is reported to be directly proportional to cell proliferation and in addition, scaffolds with rougher surface lead to improved platelet adhesion and thrombin formation [45,46], which are favorable conditions to accelerate the treatment of skin lesions. Furthermore, it has been reported that the rougher the surface of a wound dressing, the better is the entanglement between the surface of the dressing and the wound's necrotic tissue [47], which implies that when the dressing is changed, the wound is debrided, stimulating new tissue formation. The range of roughness observed for the alginate-chitosan samples varied from 1.3 to $21 \mu \mathrm{m}$ which is appropriate for the envisaged application considering 
Table 3

Thickness, roughness and color parameters of the membranes prepared in the presence of different Pluronic F68 amounts.

\begin{tabular}{|c|c|c|c|c|c|c|c|c|}
\hline \multirow[t]{2}{*}{ Samples } & \multirow[t]{2}{*}{ Thickness (mm) } & \multirow[t]{2}{*}{$R a(\mu \mathrm{m})$} & \multicolumn{6}{|c|}{ Color parameters } \\
\hline & & & $L$ & $a$ & $b$ & Hue & $C_{M}$ & $\Delta E$ \\
\hline $\mathrm{P} 0 \%$ & $0.08 \pm 0.01^{\mathrm{a}}$ & $1.3 \pm 0.1^{\mathrm{a}}$ & $96.1 \pm 0.1^{\mathrm{a}}$ & $-0.4 \pm 0.1^{\mathrm{a}}$ & $1.6 \pm 0.1^{\mathrm{a}}$ & $104 \pm 2^{\mathrm{a}}$ & $1.7 \pm 0.1^{\mathrm{a}}$ & 0 \\
\hline $\mathrm{P} 2 \%$ & $0.36 \pm 0.03^{b}$ & $18 \pm 1^{\mathrm{b}}$ & $68 \pm 2^{b}$ & $-0.6 \pm 0.1^{\mathrm{a}}$ & $3.5 \pm 0.2^{\mathrm{b}}$ & $100 \pm 1^{\mathrm{a}}$ & $3.6 \pm 0.2^{\mathrm{b}}$ & $28 \pm 2^{\mathrm{a}}$ \\
\hline P10\% & $0.50 \pm 0.04^{\mathrm{c}}$ & $21 \pm 1^{\mathrm{c}}$ & $60 \pm 1^{c}$ & $-0.6 \pm 0.1^{\mathrm{a}}$ & $3.4 \pm 0.1^{\mathrm{b}}$ & $100 \pm 1^{\mathrm{a}}$ & $3.5 \pm 0.1^{\mathrm{b}}$ & $36 \pm 1^{\mathrm{b}}$ \\
\hline
\end{tabular}

Different letters in the same column indicate significant difference at 90\% confidence limits (Tukey test).

that roughness up to $200 \mu \mathrm{m}$ are indicated in the literature for the treatment of lesions in the initial healing stages [47].

The color parameters described in Table 3 show that luminosity $(L)$ decreases when the amount of surfactant increases, which is reflected by an increase in the opacity of the membranes. The parameters $a$ and $b$ were used to calculate the tonality (Hue), which did not differ significantly among the samples. Therefore, all the membranes present similar tonality, tending to yellow. Color intensity was evaluated through the parameter chroma metric $\left(C_{M}\right)$, whose maximum value can be 16 , according to the CIELAB color metric [48]. As observed in Table 3, $C_{M}$ increased after inclusion of Pluronic F68. The difference observed for the colors of the samples prepared with and without Pluronic F68 was also very significant, as confirmed by the values calculated for the parameter $\Delta E$ (using sample $\mathrm{P} \%$ as reference). In principle there is no ideal color for a wound dressing and both transparent and opaque wound dressings are commercially available. Although high transparency permits better control and assistance, as it enables easier monitoring of the healing process, it does not imply a better functionality.

\subsection{Behavior in aqueous media}

The results obtained for the liquid uptake and mass losses of the membranes in different aqueous media are shown in Fig. 3. Data show that membranes prepared without surfactant ( $\mathrm{P0} \%$ ) presented the highest uptake in water (around $20 \mathrm{~g}$ of water per gram of membrane) and the lowest uptake in SBF and FBS media (below $5 \mathrm{~g} / \mathrm{g}$ ). Intermediate values (around $15 \mathrm{~g} / \mathrm{g}$ ) were measured in $0.9 \% \mathrm{NaCl}$ and PBS. A similar trend was observed for the membranes formulated with Pluronic F68 (P2\% and P10\%), which had higher liquid uptake in water and $\mathrm{NaCl} 0.9 \%$ followed by PBS. The lower uptake values were observed in SBF and FBS.

It is known that the ions present in all the tested fluids (with exception of water) can induce screening of free charges remaining in the polysaccharides, reducing the electrostatic repulsion between the polymeric chains, originating a packed and less hydrophilic structure with lower liquid absorption capacity [49-51]. Moreover, the ionic strength gradient in the case of the salt solutions is higher, which reduces the flux of water into the polymer matrix.

The studied samples presented, in general, higher liquid uptake values when compared to previously reported data for membranes prepared with higher Pluronic amounts and which absorbed $13.83 \mathrm{~g}$ of water, $11.96 \mathrm{~g}$ of $0.9 \% \mathrm{NaCl}$ solution, $7.74 \mathrm{~g}$ of SBF and $7.95 \mathrm{~g}$ of FBS per gram of membrane [20].

The porous samples (P2\% and P10\%) described herein presented the highest liquid uptake capacities in almost all fluids, with exception of water. Bellini et al. [7] also observed that chitosan-xanthan $(1: 1, \mathrm{w} / \mathrm{w})$ porous membranes prepared with $0.75 \%(w / w)$ Pluronic F68 had significantly lower liquid uptake in saline solutions $(0.9 \% \mathrm{NaCl}$ and $\mathrm{SBF})$ when compared to water.

After $24 \mathrm{~h}$, the amount of water absorbed by the membranes was statistically similar for all the samples. Equilibrium was reached after $20 \mathrm{~h}$ (kinetic absorption data not shown), with maximum water uptake levels that varied from 19 to $22 \mathrm{~g}_{\text {water }} / \mathrm{g}_{\text {membrane. These values are }}$ comparable to the water uptake data reported by Rodrigues et al. [19] for chitosan-alginate wound dressings, which absorbed from 11 up to
$19 \mathrm{~g}_{\text {water }} / \mathrm{g}_{\text {membrane, }}$ depending on the processing conditions of the biomaterials. On the other hand, chitosan-xanthan membranes were reported to absorb almost twice the amount of water measured in this work for chitosan-alginate porous membranes, with values that varied from 16 up to $40 \mathrm{~g}_{\text {water }} / \mathrm{g}_{\text {membrane }}$ [8]. This behavior may be explained by the different compositions of membranes produced in this work and membranes produced by Veiga and Moraes [8] together with the fact that chitosan-xanthan membranes were not cross-linked with calcium. Higher water uptake amounts were also reported by Tanodekaew et al. [52] for $\beta$-chitin and polyacrylic acid hydrogels, with values that ranged from 30 to $60 \mathrm{~g}_{\text {water }} / \mathrm{g}_{\text {membrane }}$ depending on the acrylic acid content of the hydrogels. The water uptake absorption rates of those hydrogels were also slower (equilibrium was reached only after 3 to 4 days) than for the membranes prepared in the present work (which attained the equilibrium after $20 \mathrm{~h}$ ).

When comparing the mass losses in different aqueous media (Fig. 3b) it can be observed that the membranes were less stable when immersed in water in comparison to the other solutions containing salts and other charged components (such as proteins in the case of FBS). As discussed earlier, the screening of the free charges of the polysaccharides promoted by the salt ions originates a packed structure, that
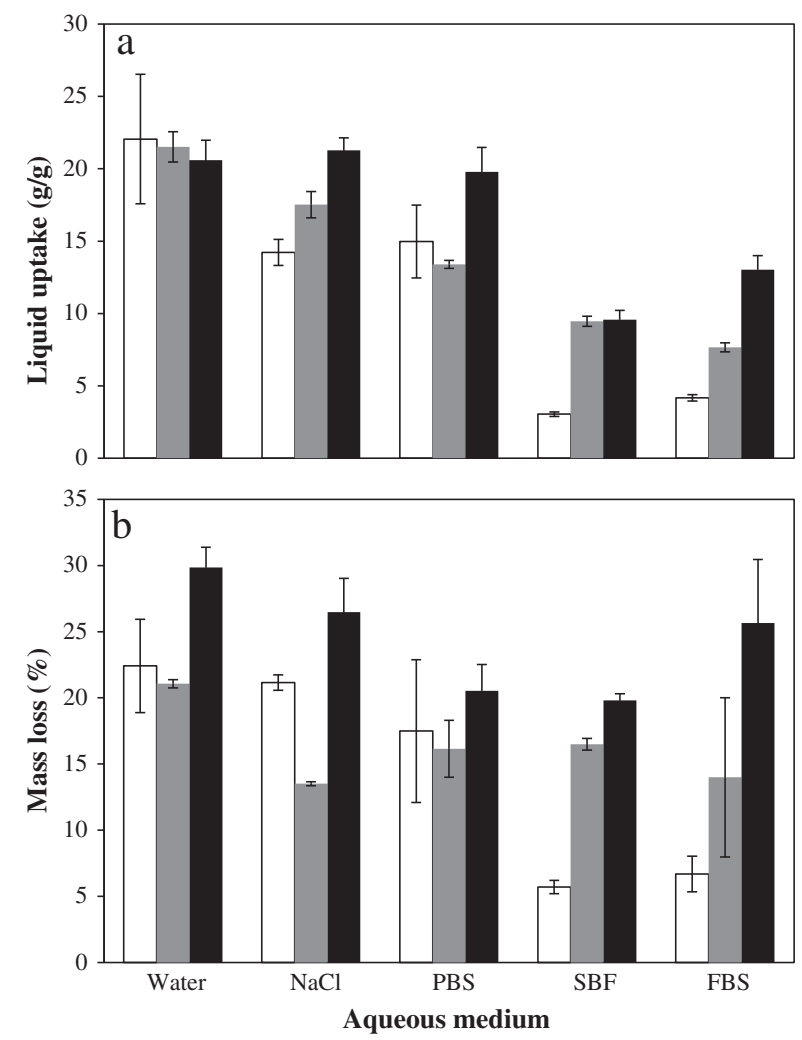

Fig. 3. Liquid uptake (a) and mass loss (b) of the membranes prepared without and with

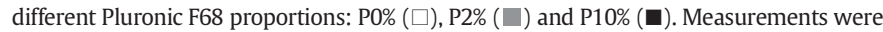
performed in different aqueous media: deionized water, $0.9 \% \mathrm{NaCl}$, phosphate buffered saline (PBS), simulated body fluid (SBF) and fetal bovine serum (FBS). 
difficult water penetration into the membranes [49-51], which consequently improves the stability of the membranes (less pronounced degradation). Moreover, mass loss results permitted us to conclude that ions from the saline solutions ( $\mathrm{NaCl}$ and PBS) do not break the $\mathrm{Ca}^{2+}$ crosslinked bonds, through cation exchange and chelation respectively, since that would lead to increased liquid uptake and membrane disintegration in those solutions, which was not observed. The sample P10\%, which has the highest porosity (0.84), presented the highest mass loss values in almost all the studied aqueous media. When compared with P0\% samples (porosity of 0.46 ), P2\% samples (porosity of 0.64 ) had higher mass losses in SBF and FBS and lower mass losses in the 0.9\% $\mathrm{NaCl}$ solution. In the remaining aqueous media both samples presented similar stability. Based on these results, sample P0\% is suitable for applications that require higher stability of the biomaterial, for example, in the case of a wound dressing that should remain in contact with the skin for a long period, while sample P2\% or P10\% should be considered if higher liquid uptake is required, for instance to efficiently absorb the exudate from a wound, or if higher porosity is needed to allow cell growth.

The mass losses in PBS, after 7 days at $37{ }^{\circ} \mathrm{C}$, varied between $\sim 16$ and $21 \%$. These values can be compared to those measured for BSA (bovine serum albumin) and BSA-casein scaffolds, which ranged between 15\% and $60 \%$ after 15 days at $37{ }^{\circ} \mathrm{C}$, depending on the sample composition and $\mathrm{pH}$ used to prepare the samples [6]. Liquid uptake values observed in that work for samples immersed in PBS at pH 7.4 for $24 \mathrm{~h}$ at $37{ }^{\circ} \mathrm{C}$ were much lower (varying from 0.075 to $0.16 \mathrm{~g}_{\text {water }} / \mathrm{g}_{\text {membrane }}$ ) than those measured in the present work (varying from 13.39 to $19.79 \mathrm{~g}_{\text {water }} / \mathrm{g}_{\text {membrane }}$ ).

Verma et al. [53] prepared chitosan-alginate PEC films with different alginate compositions (varying from 50 to $70 \%$ in weight) to be used as anti-adhesion barriers after neurosurgery. The films were obtained by drop wise addition of a chitosan solution $1 \%(\mathrm{w} / \mathrm{v})$ to an alginate solution $1 \%(\mathrm{w} / \mathrm{v})$, followed by sonication and air drying. The samples containing chitosan and alginate at the proportion of 1:1 absorbed a significantly lower amount of water $\left(1.1 \mathrm{~g}_{\text {water }} / \mathrm{g}_{\text {membrane }}\right.$ and attained equilibrium in $6 \mathrm{~h}$ ) than the ones prepared in the present work, using the same polysaccharide proportion, which could attributed to differences in the preparation procedures used in each case. Those samples were also reported to be very stable in PBS at $37^{\circ} \mathrm{C}$ after periods up to one month although mass loss results were not reported.

Hydrophilicity is another important characteristic of wound dressings and scaffolds since it directly influence cell attachment, growth and migration mechanisms [52]. However, excessive hydrophilicity may cause rapid liquid absorption and dehydration of the wound bed. In this work, water contact angle measurements were used to evaluate the surface hydrophilicity of the prepared materials and they were shown to remain almost unchanged for all the samples $\left(62 \pm 3^{\circ}\right.$, $63 \pm 3^{\circ}$ and $67 \pm 3^{\circ}$ for P0\%, P2\% and P10\%, respectively) indicating that the use of the surfactant did not significantly alter the matrixwater interactions. These results are in agreement with the liquid uptake results presented before. The membranes can be considered hydrophilic given that the obtained values are lower than $90^{\circ}$ [54]. Similar values $\left(65.2^{\circ}\right)$ were previously reported for chitosan and PVA based wound dressings [55], while higher values (varying from $73.8^{\circ}$ to $88.1^{\circ}$ ) were found for chitosan and hyaluronic acid based dressings [56].

\subsection{Mechanical properties}

The mechanical properties of the biomaterials prepared in this work are presented in Table 4 and expressed in terms of their tensile strength and elongation at break.

Results obtained for the highest proportion of surfactant show a clear decrease in the tensile strength of the membranes (up to 28 times) and on their elongation at break (up to 2 times). This decrease was expected since membranes prepared with Pluronic F68 have higher
Table 4

Tensile strength (TS) and elongation at break $(E B)$ of the membranes prepared without and with different Pluronic F68 proportions.

\begin{tabular}{lrl}
\hline Sample & TS $(\mathrm{MPa})$ & $E B(\%)$ \\
\hline P0\% & $31.1 \pm 1.7^{\mathrm{a}}$ & $4.0 \pm 0.2^{\mathrm{a}}$ \\
P2\% & $3.1 \pm 0.3^{\mathrm{b}}$ & $3.6 \pm 0.2^{\mathrm{a}}$ \\
P10\% & $1.1 \pm 0.1^{\mathrm{b}}$ & $2.0 \pm 0.1^{\mathrm{b}}$
\end{tabular}

Different letters in the same column indicate significant difference at $90 \%$ confidence limits (Tukey test).

porosity and are consequently less resistant to stress when compared to the compact deaerated films prepared without surfactant. Bellini et al. [7] also observed that chitosan-xanthan $(1: 1 \mathrm{w} / \mathrm{w})$ membranes prepared without surfactant presented significantly higher tensile strength (around $25 \mathrm{MPa}$ ) than membranes prepared with $0.75 \%(\mathrm{v} / \mathrm{w})$ of the surfactants Tween 80 (0.53 MPa) or Pluronic F68 (1.25 MPa).

The mechanical properties of the membranes obtained in the present work were superior to those previously reported for membranes prepared using the same polysaccharides and surfactant, but according to a different experimental procedure. The tensile strength and elongation at break of those membranes were equal to $0.98 \mathrm{MPa}$ and $1.96 \%$, respectively [20]. Therefore, the modifications proposed in the present work not only facilitated the production of the membranes, but also significantly improved the mechanical properties of the obtained biomaterials. Nevertheless, the obtained tensile strength and elongation at break values are still considered low for the envisaged applications, as wound dressings and scaffolds, especially if these biomaterials are intended to be used in body regions that require enhanced elasticity and mechanical resistance, such as articulations. This issue can be overcome if the membranes are hydrated before application since water will act as a plasticizer agent, able to improve the elongation at break of the samples up to 6 times, as reported by Rodrigues et al. [19].

\subsection{Indirect cytotoxicity}

The cytotoxicity of the membranes to L929 cells was analyzed via an indirect test and the obtained values were equal to $-2 \pm 8,4 \pm 2$ and $7 \pm 1 \%$ for samples $\mathrm{P} 0 \%$, P2\% and P10\%, respectively. These results show that none of the membranes negatively affects cell growth. Bellini et al. [7] also observed low cytotoxicity of chitosan-xanthan membranes to L929 cells, with values that were equal to $1.6 \%$ (without Pluronic F68) and $2.6 \%$ (for formulations prepared with $0.75 \% \mathrm{w} / \mathrm{v}$ of Pluronic F68).

Low cytotoxicity of these biomaterials was expected since both PECs obtained from both biopolymers are biocompatible and show adequate behavior in vivo when used as wound dressings [57,58] and macromolecular surfactants, such as Pluronic F68, present low toxicity and irritation effects [59], being nontoxic to P388 cells (mouse leukemic cells) in concentrations ranging from $2 \times 10^{-5}$ to $0.5 \%(\mathrm{w} / \mathrm{v})$ [60]. Even if considering that all the Pluronic F68 used in the preparation of the membranes remained in the material after the washing steps, and that all of it was extracted by the RPMI- 1640 culture medium, its final concentration in the culture medium would be equal to $0.1 \%(\mathrm{w} / \mathrm{v})$ and $0.5 \%(\mathrm{w} / \mathrm{v})$ for samples P2\% and P10\%, respectively.

\section{Conclusion}

Porous chitosan-alginate membranes, prepared using a simple and cost/time effective procedure, were characterized in this work. The membranes have excellent potential to be used in the treatment of skin lesions, and present a significant improved performance regarding liquid uptake capacities and mechanical properties when compared to previously attained results [20].

It was demonstrated that significant changes in the physicalchemical-mechanical properties of chitosan-alginate polyelectrolyte complexes can be achieved by the addition of small amounts of 
surfactant (Pluronic F68) to the polysaccharides mixture. The obtained samples present different morphologies, thicknesses (varying from 0.08 to $0.50 \mathrm{~mm}$ ), roughness (varying from 1.3 to $21 \mu \mathrm{m}$ ), porosity (varying from 0.46 to 0.84 ), mechanical properties (tensile strength from 1.1 to $31.1 \mathrm{MPa}$ and elongation at break from 2 to $4 \%$ ) and behaviors in aqueous solutions (highest liquid uptake of around $22 \mathrm{~g} / \mathrm{g}$ and mass loss of approximately 30\%), depending on the used amount of surfactant. On the other hand, properties such as the surface hydrophilicity, indirect cytotoxicity, and color were not significantly altered by the use of Pluronic F68 while the pore size dimensions do not present a clear tendency of variation with the Pluronic F68 proportion.

In general terms, all the prepared samples present favorable physicochemical and biological properties that enable their application as wound dressings and/or scaffolds, and those can be tuned, depending on the desired application, by changing the proportion of Pluronic F68 used (relatively to the total mass of biopolymers). In vivo tests are currently being performed to evaluate the performance of these biomaterials in skin lesions and the results will be reported soon.

\section{Acknowledgments}

The authors thank the grant sponsors Fundação de Amparo à Pesquisa do Estado de São Paulo (FAPESP) (2009/17755-9), Coordenação de Aperfeiçoamento de Pessoal de Nível Superior (CAPES) (288/11), Conselho Nacional de Desenvolvimento Científico e Tecnológico (CNPq) (308871/2012-0), Fundação para a Ciência e a Tecnologia (FCT, Portugal) (288/11) and Programa Iberoamericano de Ciencia y Tecnologia para el Desarrollo (CYTED) (Red 211RT0423).

\section{References}

[1] F. Khan, S.R. Ahmad, Polysaccharides and their derivatives for versatile tissue engineering application, Macromol. Biosci. 13 (4) (2013) 395-421.

[2] M. Widhe, J. Johansson, M. Hedhammar, et al., Invited review: current progress and limitations of spider silk for biomedical applications, Biopolymers 97 (6) (2012) 468-478.

[3] L.L. Hyland, M.B. Taraban, B. Hammouda, et al., Mutually reinforced multicomponent polysaccharide networks, Biopolymers 95 (12) (2011) 840-851.

[4] M. Rinaudo, Main properties and current applications of some polysaccharides as biomaterials, Polym. Int. 57 (2008) 397-430.

[5] N.S. Greaves, S.A. Iqbal, M. Baguneid, et al., The role of skin substitutes in the management of chronic cutaneous wounds, Wound Repair Regen. 21 (2) (2013) 194-210.

[6] A.J.A.M. Ribeiro, A.C. Gomes, A.M. Cavaco-Paulo, Developing scaffolds for tissue engineering using the $\mathrm{Ca}^{2+}$-induced cold gelation by an experimental design approach, J. Biomed. Mater. Res. B Appl. Biomater. 100 (8) (2012) 2269-2278.

[7] M.Z. Bellini, A.L.R. Pires, M.O. Vasconcelos, et al., Comparison of the properties of compacted and porous lamellar chitosan-xanthan membranes as dressings and scaffolds for the treatment of skin lesions, J. Appl. Polym. Sci. 125 (2012) E421-E431.

[8] I.G. Veiga, A.M. Moraes, Study of the swelling and stability properties of chitosanxanthan membranes, J. Appl. Polym. Sci. 124 (2012) E154-E160.

[9] N. Mennini, S. Furlanetto, M. Cirri, et al., Quality by design approach for developing chitosan-Ca-alginate microspheres for colon delivery of celecoxib-hydroxypropylß-cyclodextrin-PVP complex, Eur. J. Pharm. Biopharm. 80 (1) (2012) 67-75.

[10] M.A. Da Silva, A.C.K. Bierhalz, T.G. Kieckbusch, Modelling natamycin release from alginate/chitosan active films, Int. J. Food Sci. Technol. 47 (4) (2012) 740-746.

[11] I. Arzate-Vázquez, J.J. Chanona-Pérez, G. Calderón-Domínguez, et al., Microstructural characterization of chitosan and alginate films by microscopy techniques and texture image analysis, Carbohydr. Polym. 87 (1) (2012) 289-299.

[12] M. Miraftab, J. Barnabas, J.F. Kennedy, et al., Antimicrobial properties of alginatechitosan (alchite) fibers developed for wound care applications, J. Ind. Text. 40 (4) (2011) 345-360.

[13] S.J. Florczyk, D.J. Kim, D.L. Wood, et al., Influence of processing parameters on pore structure of 3D porous chitosan-alginate polyelectrolyte complex scaffolds, J. Biomed. Mater. Res. A 98 (4) (2011) 614-620.

[14] P.S. Anbinder, L. Deladino, A.S. Navarro, et al., Yerba mate extract encapsulation with alginate and chitosan systems: interactions between active compound encapsulation polymers, J. Encapsulation Adsorpt. Sci. 01 (04) (2011) 80-87.

[15] X. Yan, E. Khor, L.Y. Lim, PEC films prepared from chitosan-alginate coacervates, Chem. Pharm. Bull. 48 (7) (2000) 941-946.

[16] L. Wang, E. Khor, L.Y. Lim, Chitosan-alginate- $\mathrm{CaCl}_{2}$ system for membrane coat application, J. Pharm. Sci. 90 (8) (2001) 1134-1142.

[17] X.L. Yan, E. Khor, L.Y. Lim, Chitosan-alginate films prepared with chitosans of different molecular weights, J. Biomed. Mater. Res. 58 (4) (2001) 358-365.
[18] L. Wang, E. Khor, A. Wee, et al., Chitosan-alginate PEC membrane as a wound dressing: assessment of incisional wound healing, J. Biomed. Mater. Res. 63 (5) (2002) 610-618.

[19] A.P. Rodrigues, E.M.S. Sanchez, A.C. Costa, et al., The influence of preparation conditions on the characteristics of chitosan-alginate dressings for skin lesions, J. Appl. Polym. Sci. 109 (2008) 2703-2710.

[20] C.Z. Bueno, A.M. Moraes, Development of porous lamellar chitosan-alginate membranes: effect of different surfactants on biomaterial properties, J. Appl. Polym. Sci. 122 (2011) 624-631.

[21] G.T. Rodeheaver, L. Kurtz, B.J. Kircher, et al., Pluronic F68: a promising new skin wound cleanser, Ann. Emerg. Med. 9 (11) (1980) 572-576.

[22] M. Mancini, M. Moresi, F. Sappino, Rheological behaviour of aqueous dispersions of algal sodium alginates, J. Food Eng. 28 (1996) 283-295.

[23] A.M.N.C. Canella, R.B. Garcia, Caracterização de quitosana por cromatografia de permeação em gel - influência do método de preparação e do solvente, Quím. Nova 24 (2001) 13-17.

[24] A. Cárdenas, W. Argüelles-Monal, F.M. Goycoolea, et al., Diffusion through membranes of the polyelectrolyte complex of chitosan and alginate, Macromol. Biosci. 3 (10) (2003) 535-539.

[25] ISO 4288, Geometrical Product Specifications (GPS) - Surface Texture: Profile Method - Rules and Procedures for the Assessment of Surface Texture, 1996.

[26] ASME B46.1, Surface Texture (Surface Roughness, Waviness, and Lay), 2002.

[27] T. Kokubo, H. Kushitani, S. Sakka, et al., Solutions able to reproduce in vivo surfacestructure changes in bioactive glass-ceramic A-W3, J. Biomed. Mater. Res. 24 (1990) 721-734.

[28] ASTM D882, Standard Test Method for Tensile Properties of Thin Plastic Sheeting, 1995.

[29] ISO 10993-5e, Biological Evaluation of Medical Devices. Part 5: Tests for Cytotoxicity: In Vitro Methods, 1992.

[30] T. Wang, Y. Wu, A.J. Zeng, Synthesis and characterization of amphiphilic Pluronic copolymers and their micelles as a drug carrier, J. Appl. Polym. Sci. 117 (2010) 604-613.

[31] G.M.E. Maghraby, A.H. Alomrani, Synergistic enhancement of itraconazole dissolution by ternary system formation with Pluronic F68, Sci. Pharm. 77 (2009) 401-417.

[32] M.G. Sankalia, R.C. Mashru, J.M. Sankalia, et al., Reversed chitosan-alginate polyelectrolyte complex for stability improvement of alpha-amylase: optimization and physicochemical characterization, Eur. J. Pharm. Biopharm. 65 (2) (2007) 215-232.

[33] G. Lawrie, I. Keen, B. Drew, et al., Interactions between alginate and chitosan biopolymers characterized using FTIR and XPS, Biomacromolecules 8 (8) (2007) 2533-2541.

[34] J. Ostrowska-Czubenko, M. Gierszewska-Drużyńska, Effect of ionic crosslinking on the water state in hydrogel chitosan membranes, Carbohydr. Polym. 77 (3) (2009) 590-598.

[35] P. Kan, Z.B. Chen, R.Y. Kung, et al., Study on the formulation of o/w emulsion as carriers for lipophilic drugs, Colloids Surf. B 15 (1999) 117-125.

[36] W.L.J. Hinrichs, E.J.C.M.P. Lommen, R.H. Wildevuur, et al., Fabrication and characterization of an asymmetric polyurethane membrane for use as a wound dressing J. Appl. Biomater. 3 (1992) 287-303.

[37] K. Holmberg, B. Jönsson, B. Kronberg, et al., Surfactants and Polymers in Aqueous Solutions, 2nd ed. John Wiley \& Sons, Chichester, 2002.

[38] W.L. Stoppel, J.C. White, S.D. Horava, et al., Transport of biological molecules in surfactant-alginate composite hydrogels, Acta Biomater. 7 (11) (2011) 3988-3998.

[39] Y. Chisti, Animal-cell damage in sparged bioreactors, Trends Biotechnol. 18 (10) (2000) 420-432.

[40] F. Quignard, R. Valentin, F. Di Renzo, Aerogel materials from marine polysaccharides, New J. Chem. 32 (8) (2008) 1300-1310.

[41] G.H. Kim, H. Yoon, A direct-electrospinning process by combined electric field and air-blowing system for nanofibrous wound-dressings, Appl. Phys. A 90 (3) (2008) 389-394.

[42] D. Brahatheeswaran, A. Mathew, R.G. Aswathy, et al., Hybrid fluorescent curcumin loaded zein electrospun nanofibrous scaffold for biomedical applications, Biomed. Mater. 7 (4) (2012) 1-16.

[43] J. Ma, H. Wang, B. He, et al., A preliminary in vitro study on the fabrication and tissue engineering applications of a novel chitosan bilayer material as a scaffold of human neofetal dermal fibroblasts, Biomaterials 22 (2001) 331-336.

[44] I. Adekogbe, A. Ghanem, Fabrication and characterization of DTBP-crosslinked chitosan scaffolds for skin tissue engineering, Biomaterials 26 (35) (2005) 7241-7250.

[45] A.F. Von Recum, C.E. Shannon, C.E. Cannon, et al., Modifiers of cellular adhesion, J. Tissue Eng. 2 (4) (1996) 241-253.

[46] V. Milleret, T. Hefti, H. Hall, et al., Influence of the fiber diameter and surface roughness of electrospun vascular grafts on blood activation, Acta Biomater. 8 (12) (2012) 4349-4356.

[47] Boehringer JR, Karpowicz J, Mitra A, et al. Growth Stimulating Wound Dressing With Improved Contact Surfaces. Patent US.7951124 B2, USA, 2011.

[48] Konica Minolta Inc., Precise Color Communication - Color Control From Perception to Instrumentation, 2007. (Japan).

[49] G. Maurstad, Y.A. Morch, A.R. Bausch, et al., Polyelectrolyte layer interpenetration and swelling of alginate-chitosan multilayers studied by dual wavelength reflection interference contrast microscopy, Carbohydr. Polym. 71 (4) (2008) 672-681.

[50] A. Bartkowiak, Effect of the ionic strength on properties of binary alginate/ oligochitosan microcapsules, Colloids Surf. A Physicochem. Eng. Asp. 204 (1-3) (2002) 117-124.

[51] S.P. Strand, M.S. Vandvik, K.M. Vårum, et al., Screening of chitosans and conditions for bacterial flocculation, Biomacromolecules 2 (1) (2001) 126-133.

[52] S. Tanodekaew, M. Prasitsilp, S. Swasdison, et al., Preparation of acrylic grafted chitin for wound dressing application, Biomaterials 25 (7-8) (2004) 1453-1460. 
[53] D. Verma, M.L. Previtera, R. Schloss, et al., Polyelectrolyte complex membranes for prevention of post-surgical adhesions in neurosurgery, Ann. Biomed. Eng. 40 (9) (2012) 1949-1960

[54] R. Förch, H. Schönherr, T.A. Jenkins, Surface Design: Applications in Bioscience and Nanotechnology, 1st ed. Wiley-VCH, Weinheim, 2009.

[55] Y.O. Kang, I.S. Yoon, S.Y. Lee, et al., Chitosan-coated poly(vinyl alcohol) nanofibers for wound dressings, J. Biomed. Mater. Res. B Appl. Biomater. 92 (2) (2010) 568-576.

[56] H. Xu, L. Ma, H. Shi, et al., Chitosan-hyaluronic acid hybrid film as a novel wound dressing: in vitro and in vivo studies, Polym. Adv. Technol. 18 (2007) 869-875.
[57] W. Paul, C.P. Sharma, Chitosan and alginate wound dressings: a short review, Trends Biomater. Artif. Organs 18 (1) (2004) 18-23.

[58] N.M. Paulo, M. Conceição, I.A. Bueno, M.S.B. Silva, L.B. Menezes, A.M. Moraes, A.P. Rodrigues, Chitosan film for treatment of cutaneous wound in a female cat, Acta Sci. Vet. 35 (3) (2007) 381-383.

[59] T.F. Tadros, Applied Surfactants: Principles and Applications, 1st ed. Wiley-VCH, Berkshire, 2005.

[60] K. Bogman, F. Erne-Brand, J. Alsenz, et al., The role of surfactants in the reversal of active transport mediated by multidrug resistance proteins, J. Pharm. Sci. 92 (6) (2003) 1250-1261. 